\title{
The construction of several new inequalities for definite integral
}

\author{
Hua-Sheng Zheng ${ }^{\mathrm{a}}$, Wan-Yuan Ming ${ }^{\mathrm{a}, *}$, Da-Ming Yuan ${ }^{\mathrm{b}}$ \\ a School of Mathematics and Information Sciences, Nanchang Hangkong University, \\ Nanchang 330063 China \\ b School of Mathematics and Statistics, Jiangxi Normal University, Nanchang 330022 China
}

*Corresponding author, e-mail: mingwanyuan@sina.com

Received 17 Nov 2020

Accepted 14 Jun 2021

\begin{abstract}
In this paper, a series of new inequalities for definite integral are constructed. By rigorous analysis, the sufficient and necessary conditions for which the equality holds are carried out. Additionally, the optimal constant factors for these new inequalities are obtained.
\end{abstract}

KEYWORDS: integral inequality, Cauchy-Schwarz inequality, optimal constant factor

MSC2010: 26D06

\section{INTRODUCTION}

The theory of inequalities began its development from the time when Gauss, Cauchy and Čebyšev established the theoretical foundation for approximative methods [1]. At present, inequalities play an crucial important role in all fields of mathematics and are studied extensively: in some of them classical inequalities were sharpened or generalized, in some other works new inequalities were discovered, and also in some papers the connection of these famous inequalities are investigated. For instance, the Cauchy-Schwarz inequality is a useful inequality encountered in many different settings, such as linear algebra applied to vectors, in analysis applied to infinite series and integration of products, for details we refer to $[1,2]$. The discrete and continuous Gronwall-Bellman inequalities are often used in the analysis of existence, boundedness, stability of numerical solutions of differential equations and integral equations [3-6]. In 2005, Yang et al [7] established an extension on Hardy-Hilbert integral inequality by introducing a power exponent function, and show the best possible coefficient. In 2014, Gu et al [8] improved the upper and lower bounds for the I. Schur inequality, and obtained some new I. Schur inequality. As to the mathematical equivalence among some famous inequalities, Li et al [9] presented several generalization of the Radon inequality, and proved the equivalence relation of the weighted power mean inequality and
Radon inequality. Additionally, in 2018, Li et al [10] further discussed the mathematical equivalence of the weighted arithmetic mean-geometric mean inequality, Hölder inequality and the weighted powermean inequality. In this work, we will concern with the methods of how to construct new inequalities for definite integral, and investigate the best possible coefficient for these new inequalities.

\section{MAIN RESULTS}

In this section, we will construct some new integral inequalities associated with $\left(\int_{a}^{b}(x-\xi)^{n} f(x) \mathrm{d} x\right)^{2}$ and $\int_{a}^{b}\left(f^{(k)}(x)\right)^{2} \mathrm{~d} x$ for $\xi=0, a, b$ and $(a+b) / 2$, respectively.

The inequalities related to $\left(\int_{a}^{b} x^{n} f(x) \mathrm{d} x\right)^{2}$ and $\int_{a}^{b}\left(f^{(k)}(x)\right)^{2} \mathrm{~d} x$

Theorem 1 Suppose $f(x) \in C^{1}[a, b]$ and $f(a)=$ $f(b)=0$, then

$$
\left(\int_{a}^{b} x f(x) \mathrm{d} x\right)^{2} \leqslant M_{1} \int_{a}^{b}\left(f^{\prime}(x)\right)^{2} \mathrm{~d} x
$$

where

$$
M_{1}=\frac{(b-a)-\frac{2}{3} s\left(b^{3}-a^{3}\right)+\frac{s^{2}}{5}\left(b^{5}-a^{5}\right)}{4 s^{2}}
$$

and $s$ is a non-zero constant. Furthermore, 
(i) the constant factor $M_{1}$ is optimal if and only if $s=3(b-a) /\left(b^{3}-a^{3}\right)$, and this value is

$$
M_{1}^{\mathrm{opt}}=\frac{b^{5}-a^{5}}{20}-\frac{1}{36} \frac{\left(b^{3}-a^{3}\right)^{2}}{b-a} ;
$$

(ii) when $s \neq 3(b-a) /\left(b^{3}-a^{3}\right)$, the equality holds in (1) if and only if $f(x) \equiv 0$;

(iii) when $s=3(b-a) /\left(b^{3}-a^{3}\right)$, the equality holds in (1) if and only if $f(x)=\mu(x-a)$ $\mu \frac{b-a}{b^{3}-a^{3}}\left(x^{3}-a^{3}\right)$, where $\mu$ is an arbitrary constant.

Proof: Using the integration by parts formula, together with the given conditions $f(a)=f(b)=0$, one may get

$$
\int_{a}^{b} x f(x) \mathrm{d} x=-\frac{1}{2} \int_{a}^{b} x^{2} f^{\prime}(x) \mathrm{d} x .
$$

Thus, for any $s \neq 0, s \in \mathbb{R}$, it holds

$$
\begin{aligned}
2 s \int_{a}^{b} x f(x) \mathrm{d} x & =-s \int_{a}^{b} x^{2} f^{\prime}(x) \mathrm{d} x \\
& =\int_{a}^{b}\left(1-s x^{2}\right) f^{\prime}(x) \mathrm{d} x .
\end{aligned}
$$

Applying the Cauchy-Schwarz inequality leads to

$$
\begin{aligned}
\left(\int_{a}^{b} x f(x) \mathrm{d} x\right)^{2} & =\left(\frac{\int_{a}^{b}\left(1-s x^{2}\right) f^{\prime}(x) \mathrm{d} x}{2 s}\right)^{2} \\
& \leqslant \frac{\int_{a}^{b}\left(1-s x^{2}\right)^{2} \mathrm{~d} x}{4 s^{2}} \int_{a}^{b}\left(f^{\prime}(x)\right)^{2} \mathrm{~d} x \\
& =M_{1} \int_{a}^{b}\left(f^{\prime}(x)\right)^{2} \mathrm{~d} x,
\end{aligned}
$$

where $M_{1}$ is defined in Theorem 1 .

Recalling that in the above process of adopting the Cauchy-Schwarz inequality, the equality holds in (1) if and only if the functions $f^{\prime}(x)$ and $\left(1-s x^{2}\right)$ are linear dependent, i.e., $f^{\prime}(x)=\mu\left(1-s x^{2}\right)$. Therefore $f(x)=\mu x-\frac{\mu}{3} s x^{3}+C$. Substituting $f(a)=f(b)=0$ in above expression of $f(x)$ we may obtain

$$
\mu a-\frac{\mu}{3} s a^{3}+C=0, \quad \mu b-\frac{\mu}{3} s b^{3}+C=0 .
$$

The determinant of the coefficient matrix of (2) is

$$
D=\left|\begin{array}{ll}
a-\frac{s}{3} a^{3} & 1 \\
b-\frac{s}{3} b^{3} & 1
\end{array}\right|=a-\frac{s}{3} a^{3}-b+\frac{s}{3} b^{3} .
$$

Therefore, (i) when $D \neq 0$, i.e., $s \neq 3(b-a) /\left(b^{3}-a^{3}\right)$, (2) possesses a unique solution $\mu=C=0$, and in this case $f(x) \equiv 0$;

(ii) when $D=0$, i.e., $s=3(b-a) /\left(b^{3}-a^{3}\right)$, (2) possesses infinite solutions which are given by $C=-\mu a+\mu \frac{b-a}{b^{3}-a^{3}} a^{3}$, and in this case $f(x)=$ $\mu(x-a)-\mu \frac{b-a}{b^{3}-a^{3}}\left(x^{3}-a^{3}\right)$.

Now, we are in a position to discuss the optimal constant factor for inequality (1). Denote

$$
g(s) \triangleq M_{1}=\frac{(b-a)-\frac{2}{3} s\left(b^{3}-a^{3}\right)+\frac{s^{2}}{5}\left(b^{5}-a^{5}\right)}{4 s^{2}},
$$

then it follows from $g^{\prime}(s)=\frac{\frac{1}{3} s\left(b^{3}-a^{3}\right)-(b-a)}{2 s^{3}}=0$ that $s=\frac{3}{a^{2}+a b+b^{2}} \triangleq s_{0}$. Therefore, $g^{\prime}(s)<0$ when $s \in$ $\left(0, s_{0}\right)$, and $g^{\prime}(s)>0$ when $s \in(-\infty, 0) \bigcup\left(s_{0}, \infty\right)$. Observing that $\lim _{s \rightarrow 0} g(s)=\infty, \lim _{s \rightarrow \infty} g(s)=$ $\frac{1}{20}\left(b^{5}-a^{5}\right)$ and

$g\left(s_{0}\right)=\frac{-(b-a)}{36\left(\frac{1}{a^{2}+a b+b^{2}}\right)^{2}}+\frac{1}{20}\left(b^{5}-a^{5}\right)<\frac{1}{20}\left(b^{5}-a^{5}\right)$, we can deduce that when $s=s_{0}$, the value of $g(s)$ is minimum, and thus the optimal constant factor is

$$
M_{1}^{\mathrm{opt}}=g\left(s_{0}\right)=\frac{b^{5}-a^{5}}{20}-\frac{1}{36} \frac{\left(b^{3}-a^{3}\right)^{2}}{b-a} .
$$

The proof is then completed.

For special values $a=0$ and $b=1$, the optimal constant factor $M_{1}$ in Theorem 1 can be given explicitly which is shown in the following remark.

Remark 1 Setting $a=0$ and $b=1$ in inequality (1), it follows that when $s=3, M_{1}$ is optimal and this inequality reduces to

$$
\left(\int_{0}^{1} x f(x) \mathrm{d} x\right)^{2} \leqslant \frac{1}{45} \int_{0}^{1}\left(f^{\prime}(x)\right)^{2} \mathrm{~d} x,
$$

with the equality holding if and only if $f(x)=\mu x-$ $\mu x^{3}$ for $\mu \in \mathbb{R}$.

Theorem 2 Assume $f(x) \in C^{2}[a, b], f(a)=f(b)=$ 0 , and $f^{\prime}(a)=f^{\prime}(b)=0$, then

$$
\left(\int_{a}^{b} x f(x) \mathrm{d} x\right)^{2} \leqslant M_{2} \int_{a}^{b}\left(f^{\prime \prime}(x)\right)^{2} \mathrm{~d} x
$$

where

$$
M_{2}=\frac{(b-a)+\frac{s}{2}\left(b^{4}-a^{4}\right)+\frac{s^{2}}{7}\left(b^{7}-a^{7}\right)}{36 s^{2}},
$$

and $s$ is a non-zero constant. Furthermore, 
(i) the constant factor $M_{2}$ is optimal if and only if $s=-4(b-a) /\left(b^{4}-a^{4}\right)$, and this value is $M_{2}^{\mathrm{opt}}=\frac{b^{7}-a^{7}}{252}-\frac{1}{576} \frac{\left(b^{4}-a^{4}\right)^{2}}{b-a} ;$

(ii) when $s \neq-\frac{4(b-a)}{b^{4}-a^{4}}$, or $s=-\frac{4(b-a)}{b^{4}-a^{4}}$ and $b \neq 5 a / 3$, the equality holds in (3) if and only if $f(x) \equiv 0$;

(iii) when $s=-4(b-a) /\left(b^{4}-a^{4}\right)$ and $b=5 a / 3$, the equality holds in (3) if and only if $f(x)=$ $\frac{\mu}{2} x^{2}-\frac{\mu}{5} \frac{b-a}{b^{4}-a^{4}} x^{5}+C_{1} x+C_{2}$, where $\left(\mu, C_{1}, C_{2}\right)^{T}$ solves the following equation (6).

Proof: Observing the given conditions $f(a)=$ $f(b)=0$ and $f^{\prime}(a)=f^{\prime}(b)=0$, it follows

$$
\int_{a}^{b} x f(x) \mathrm{d} x=-\frac{1}{2} \int_{a}^{b} x^{2} f^{\prime}(x) \mathrm{d} x=\frac{1}{6} \int_{a}^{b} x^{3} f^{\prime \prime}(x) \mathrm{d} x .
$$

Therefore, for $s \neq 0, s \in \mathbb{R}$, it holds

$$
\begin{aligned}
6 s \int_{a}^{b} x f(x) \mathrm{d} x & =s \int_{a}^{b} x^{3} f^{\prime \prime}(x) \mathrm{d} x \\
& =\int_{a}^{b}\left(1+s x^{3}\right) f^{\prime \prime}(x) \mathrm{d} x
\end{aligned}
$$

Resorting to the Cauchy-Schwarz integral inequality, one may obtain

$$
\begin{aligned}
\left(\int_{a}^{b} x f(x) \mathrm{d} x\right)^{2} & =\left(\frac{\int_{a}^{b}\left(1+s x^{2}\right) f^{\prime \prime}(x) \mathrm{d} x}{6 s}\right)^{2} \\
& \leqslant \frac{\int_{a}^{b}\left(1+s x^{3}\right)^{2} \mathrm{~d} x}{36 s^{2}} \int_{a}^{b}\left(f^{\prime \prime}(x)\right)^{2} \mathrm{~d} x \\
& =M_{2} \int_{a}^{b}\left(f^{\prime \prime}(x)\right)^{2} \mathrm{~d} x,
\end{aligned}
$$

where $M_{2}$ is defined in Theorem 2. Setting

$$
h(s) \triangleq M_{2}=\frac{(b-a)+\frac{s}{2}\left(b^{4}-a^{4}\right)+\frac{s^{2}}{7}\left(b^{7}-a^{7}\right)}{36 s^{2}},
$$

then from $h^{\prime}(s)=-\frac{b-a}{18 s^{3}}-\frac{1}{72 s^{2}}\left(b^{4}-a^{4}\right)=0$ we get $s_{1}=-4(b-a) /\left(b^{4}-a^{4}\right)$. Therefore, $h^{\prime}(s)>$ 0 when $s \in\left(s_{1}, 0\right)$, and $h^{\prime}(s)<0$ when $s \in$ $\left(-\infty, s_{1}\right) \bigcup(0, \infty)$. Noting that $\lim _{s \rightarrow 0} h(s)=\infty$ and $\lim _{s \rightarrow \infty} h(s)=\left(b^{7}-a^{7}\right) / 252$, it follows that when $s=s_{1}, h(s)$ reaches the minimum value

$$
M_{2}^{\mathrm{opt}}=h\left(s_{1}\right)=\frac{b^{7}-a^{7}}{252}-\frac{1}{576} \frac{\left(b^{4}-a^{4}\right)^{2}}{b-a} .
$$

Similarly, the equality holds in (3) if and only if $f^{\prime \prime}(x)=\mu\left(1+s x^{3}\right)$, thus $f^{\prime}(x)=\mu x+\frac{\mu}{4} s x^{4}+C_{1}$ and $f(x)=\frac{\mu}{2} x^{2}+\frac{\mu}{20} s x^{5}+C_{1} x+C_{2}$. Recalling that $f^{\prime}(a)=f^{\prime}(b)=0$, we have

$$
\mu a+\frac{\mu}{4} s a^{4}+C_{1}=0, \quad \mu b+\frac{\mu}{4} s b^{4}+C_{1}=0 .
$$

Substituting $f(a)=f(b)=0$ in above expression of $f(x)$, we get

$$
\left\{\begin{array}{l}
\frac{1}{2} \mu a^{2}+\frac{\mu}{20} s a^{5}+C_{1} a+C_{2}=0 \\
\frac{1}{2} \mu b^{2}+\frac{\mu}{20} s b^{5}+C_{1} b+C_{2}=0 .
\end{array}\right.
$$

The determinant of the coefficient matrix of (4) is

$$
D=\left|\begin{array}{ll}
a+\frac{s}{4} a^{4} & 1 \\
b+\frac{s}{4} b^{4} & 1
\end{array}\right|=a+\frac{s}{4} a^{4}-b-\frac{s}{4} b^{4} .
$$

Therefore,

(i) when $D \neq 0$, i.e., $s \neq-4(b-a) /\left(b^{4}-a^{4}\right)$, (4) only possesses trivial solution $\mu=C_{1}=0$. Together with linear system (5) we get $C_{2}=0$ and in this case $f(x) \equiv 0$;

(ii) when $D=0$, i.e., $s=-4(b-a) /\left(b^{4}-a^{4}\right)$, (4) possesses infinite solutions, and thus the two equations in system (4) are linear dependent.

Observing that the parameters $\mu, C_{1}$ and $C_{2}$ should satisfy both systems (4) and (5), thus we substitute $s=-4(b-a) /\left(b^{4}-a^{4}\right)$ in these two linear systems and get

$$
\left\{\begin{array}{r}
\left(\frac{1}{2} a^{2}-\frac{1}{5} \frac{b-a}{b^{4}-a^{4}} a^{5}\right) \mu+C_{1} a+C_{2}=0 \\
\left(\frac{1}{2} b^{2}-\frac{1}{5} \frac{b-a}{b^{4}-a^{4}} b^{5}\right) \mu+C_{1} b+C_{2}=0 \\
\left(a-\frac{b-a}{b^{4}-a^{4}} a^{4}\right) \mu+C_{1}=0 .
\end{array}\right.
$$

The determinant of the coefficient matrix of (6) is

$$
D_{1}=\left|\begin{array}{lll}
\frac{a^{2}}{2}-\frac{a^{5}}{5} \frac{b-a}{b^{4}-a^{4}} & a & 1 \\
\frac{b^{2}}{2}-\frac{b^{5}}{5} \frac{b-a}{b^{4}-a^{4}} & b & 1 \\
a-\frac{b-a}{b^{4}-a^{4}} a^{4} & 1 & 0
\end{array}\right|=\frac{(b-a)(3 b-5 a)}{10},
$$

(a) when $3 b-5 a \neq 0, D_{1} \neq 0$, and (6) only possesses trivial solution $\mu=C_{1}=C_{2}=0$, and in this case $f(x) \equiv 0$;

(b) when $3 b-5 a=0, D_{1}=0$, and (6) possesses infinite solutions. In this case, $f(x)=\frac{\mu}{2} x^{2}-$ $\frac{\mu}{5} \frac{b-a}{b^{4}-a^{4}} x^{5}+C_{1} x+C_{2}$. 
Remark 2 Let $a=0$ and $b=1$ in the inequality (3), then it follows that when $k=-4, M_{2}=1 / 448$ is optimal and this inequality is given by

$$
\left(\int_{0}^{1} x f(x) \mathrm{d} x\right)^{2} \leqslant \frac{1}{448} \int_{0}^{1}\left(f^{\prime \prime}(x)\right)^{2} \mathrm{~d} x .
$$

Following similar lines we can deduce the following inequality associated with $\left(\int_{a}^{b} x^{n} f(x) \mathrm{d} x\right)^{2}$ and $\int_{a}^{b}\left(f^{(k)}(x)\right)^{2} \mathrm{~d} x$ for any $n, k \in \mathbb{N}$, which is made precise as follows.

Theorem 3 Suppose $f(x) \in C^{k}[a, b], k \geqslant 1$ and $f^{(l)}(a)=f^{(l)}(b)=0(l=0,1, \ldots, k-1)$, then for any $n \in \mathbb{N}$,

$$
\left(\int_{a}^{b} x^{n} f(x) \mathrm{d} x\right)^{2} \leqslant M_{n, k} \int_{a}^{b}\left(f^{(k)}(x)\right)^{2} \mathrm{~d} x
$$

with the equality holds if and only if $f(x)$ subjects to

$$
\begin{aligned}
f^{(k)}(x) & =\mu\left(1+s x^{n+k}\right), \\
f^{(l)}(a) & =f^{(l)}(b)=0, \quad l=0,1, \ldots, k-1,
\end{aligned}
$$

where

$$
\begin{aligned}
M_{n, k}=\frac{(b-a)+\frac{2 s}{n+k+1}\left(b^{n+k+1}-a^{n+k+1}\right)}{[(n+1)(n+2) \cdots(n+k) s]^{2}} & \\
& +\frac{\frac{s^{2}}{2 n+2 k+1}\left(b^{2 n+2 k+1}-a^{2 n+2 k+1}\right)}{[(n+1)(n+2) \cdots(n+k) s]^{2}},
\end{aligned}
$$

$s \neq 0$, and $\mu$ is an arbitrary constant.

Remark 3 For $n=2, a=0$ and $b=1$ in Theorem 3, the constant factor $M_{2,1}=1 / 112$ is optimal when $s=4$, and this inequality assumes the form

$$
\left(\int_{0}^{1} x^{2} f(x) \mathrm{d} x\right)^{2} \leqslant \frac{1}{112} \int_{0}^{1}\left(f^{\prime}(x)\right)^{2} \mathrm{~d} x .
$$

The following corollaries are direct results of Theorem 3, and the sufficient and necessary conditions for which the equality holds can be addressed precisely for these special cases.

Corollary 1 Suppose $f(x) \in C^{1}[a, b]$ and $f(a)=$ $f(b)=0$, then for any $n \in \mathbb{N}$,

$$
\left(\int_{a}^{b} x^{n} f(x) \mathrm{d} x\right)^{2} \leqslant M_{n, 1} \int_{a}^{b}\left(f^{\prime}(x)\right)^{2} \mathrm{~d} x,
$$

with the equality holding if and only if $f(x) \equiv 0$ or $f(x)=\mu(x-a)-\mu \frac{b-a}{b^{n+2}-a^{n+2}}\left(x^{n+2}-a^{n+2}\right)$, where

$M_{n, 1}=\frac{(b-a)-\frac{2 s}{n+2}\left(b^{n+2}-a^{n+2}\right)+\frac{s^{2}}{2 n+3}\left(b^{2 n+3}-a^{2 n+3}\right)}{(n+1)^{2} s^{2}}$, and $s$ is a non-zero constant. Furthermore, when $s=(n+2)(b-a) /\left(b^{n+2}-a^{n+2}\right), M_{n, 1}$ is the optimal constant factor.

Corollary 2 Suppose $f(x) \in C^{2}[a, b], \quad f(a)=$ $f(b)=0$, and $f^{\prime}(a)=f^{\prime}(b)=0$, then for any $n \in \mathbb{N}$,

$$
\left(\int_{a}^{b} x^{n} f(x) \mathrm{d} x\right)^{2} \leqslant M_{n, 2} \int_{a}^{b}\left(f^{\prime \prime}(x)\right)^{2} \mathrm{~d} x,
$$

with the equality holding if and only if $f(x) \equiv 0$ or $f(x)=\frac{\mu}{2} x^{2}-\frac{\mu}{n+4} \frac{b-a}{b^{n+3}-a^{n+3}} x^{n+4}+C_{1} x+C_{2}$, where

$M_{n, 2}=\frac{\left.(b-a)+\frac{2 s}{n+3}\left(b^{n+3}-a^{n+3}\right)\right)+\frac{s^{2}}{2 n+5}\left(b^{2 n+5}-a^{2 n+5}\right)}{(n+1)^{2}(n+2)^{2} s^{2}}$, $s$ is a non-zero constant, and $\mu, C_{1}, C_{2}$ satisfy the equations

$$
\left\{\begin{array}{r}
\left(\frac{1}{2} a^{2}-\frac{1}{n+4} \frac{b-a}{b^{n+3}-a^{n+3}} a^{n+4}\right) \mu+C_{1} a+C_{2}=0 \\
\left(\frac{1}{2} b^{2}-\frac{1}{n+4} \frac{b-a}{b^{n+3}-a^{n+3}} b^{n+4}\right) \mu+C_{1} b+C_{2}=0 \\
\left(a-\frac{b-a}{b^{n+3}-a^{n+3}} a^{n+3}\right) \mu+C_{1}=0 .
\end{array}\right.
$$

Moreover, $M_{n, 2}$ is optimal when $s=-\frac{(n+3)(b-a)}{b^{n+3}-a^{n+3}}$.

The inequalities related to $\left(\int_{a}^{b}(x-a)^{n} f(x) \mathrm{d} x\right)^{2}$ and $\int_{a}^{b}\left(f^{(k)}(x)\right)^{2} \mathrm{~d} x$

Theorem 4 Suppose $f(x) \in C^{1}[a, b]$ and $f(b)=0$, then for any $n \in \mathbb{N}$,

$$
\left(\int_{a}^{b}(x-a)^{n} f(x) \mathrm{d} x\right)^{2} \leqslant \tilde{M}_{n, 1} \int_{a}^{b}\left(f^{\prime}(x)\right)^{2} \mathrm{~d} x,
$$

with the equality holding if and only if $f(x)=f(a)-$ $f(a)\left(\frac{x-a}{b-a}\right)^{n+2}$, where $\widetilde{M}_{n, 1}=\frac{(b-a)^{2 n+3}}{(n+1)^{2}(2 n+3)}$.

Proof: Applying the integration by parts formula, and observing that $f(b)=0$, it then follows

$$
\int_{a}^{b}(x-a)^{n} f(x) \mathrm{d} x=-\frac{1}{n+1} \int_{a}^{b}(x-a)^{n+1} f^{\prime}(x) \mathrm{d} x .
$$

Using the Cauchy-Schwarz integral inequality leads to

$$
\begin{aligned}
\left(\int_{a}^{b}(x-a)^{n} f(x) d x\right)^{2} & =\left(-\frac{1}{n+1} \int_{a}^{b}(x-a)^{n+1} f^{\prime}(x) \mathrm{d} x\right)^{2} \\
& \leqslant \frac{\int_{a}^{b}(x-a)^{2(n+1)} \mathrm{d} x}{(n+1)^{2}} \int_{a}^{b}\left(f^{\prime}(x)\right)^{2} \mathrm{~d} x \\
& =\frac{(b-a)^{2 n+3}}{(n+1)^{2}(2 n+3)} \int_{a}^{b}\left(f^{\prime}(x)\right)^{2} \mathrm{~d} x \\
& =\tilde{M}_{n, 1} \int_{a}^{b}\left(f^{\prime}(x)\right)^{2} \mathrm{~d} x
\end{aligned}
$$


where $\widetilde{M}_{n, 1}$ is defined in Theorem 4. Furthermore, the equality holds in above expression if and only if $f^{\prime}(x)=\mu(x-a)^{n+1}$, that is, $f(x)=\frac{\mu}{n+2}(x-a)^{n+2}+C$. From $f(a)=C$ and $f(b)=0$ one may easily obtain $f(x)=f(a)-f(a)\left(\frac{x-a}{b-a}\right)^{n+2}$.

More generally, for any $n, k \in \mathbb{N}$, the results related to $\left(\int_{a}^{b}(x-a)^{n} f(x) \mathrm{d} x\right)^{2}$ and $\int_{a}^{b}\left(f^{(k)}(x)\right)^{2} \mathrm{~d} x$ can be stated as the following theorem.

Theorem 5 Suppose $f(x) \in C^{k}[a, b], k \geqslant 1$, and $f(b)=f^{\prime}(b)=\cdots=f^{(k-1)}(b)=0$, then for any $n \in \mathbb{N}$, it holds

$$
\left(\int_{a}^{b}(x-a)^{n} f(x) \mathrm{d} x\right)^{2} \leqslant \tilde{M}_{n, k} \int_{a}^{b}\left(f^{(k)}(x)\right)^{2} \mathrm{~d} x,
$$

with the equality holding if and only if

$$
\begin{aligned}
f^{(k)}(x) & =\mu(x-a)^{n+k}, \\
f(b) & =f^{\prime}(b)=\cdots=f^{(k-1)}(b)=0,
\end{aligned}
$$

where $\mu$ is an arbitrary constant, and

$$
\widetilde{M}_{n, k}=\frac{(b-a)^{2 n+2 k+1}}{(n+1)^{2}(n+2)^{2} \cdots(n+k)^{2}(2 n+2 k+1)} .
$$

Corollary 3 Suppose $f(x) \in C^{2}[a, b]$ and $f(b)=$ $f^{\prime}(b)=0$, then for any $n \in \mathbb{N}$,

$$
\left(\int_{a}^{b}(x-a)^{n} f(x) \mathrm{d} x\right)^{2} \leqslant \widetilde{M}_{n, 2} \int_{a}^{b}\left(f^{\prime \prime}(x)\right)^{2} \mathrm{~d} x,
$$

with the equality holding if and only if

$f(x)=\frac{f(a)}{n+3}\left(\frac{x-a}{b-a}\right)^{n+4}+f(a)\left(\frac{b-x}{b-a}-\frac{1}{n+3} \frac{x-a}{b-a}\right)$, where $\widetilde{M}_{n, 2}=(b-a)^{2 n+5} /(n+1)^{2}(n+2)^{2}(2 n+5)$.

The inequalities related to $\left(\int_{a}^{b}(x-b)^{n} f(x) \mathrm{d} x\right)^{2}$ and $\int_{a}^{b}\left(f^{(k)}(x)\right)^{2} d x$

Theorem 6 Suppose $f(x) \in C^{k}[a, b], k \geqslant 1$, and $f(a)=f^{\prime}(a)=\cdots=f^{(k-1)}(a)=0$, then for any $n \in \mathbb{N}$,

$$
\left(\int_{a}^{b}(x-b)^{n} f(x) \mathrm{d} x\right)^{2} \leqslant \hat{M}_{n, k} \int_{a}^{b}\left(f^{(k)}(x)\right)^{2} \mathrm{~d} x,
$$

with the equality holding if and only if

$$
\begin{aligned}
f^{(k)}(x) & =\mu(x-b)^{n+k}, \\
f(a) & =f^{\prime}(a)=\cdots=f^{(k-1)}(a)=0,
\end{aligned}
$$

where $\mu$ is an arbitrary constant, and

$$
\hat{M}_{n, k}=\frac{(b-a)^{2 n+2 k+1}}{(n+1)^{2}(n+2)^{2} \cdots(n+k)^{2}(2 n+2 k+1)} .
$$

Proof: The main steps of the proof are similar to the ones of Theorem 4, thus the details is omitted here.

Corollary 4 Suppose $f(x) \in C^{1}[a, b]$ and $f(a)=0$, then for any $n \in \mathbb{N}$,

$$
\left(\int_{a}^{b}(x-b)^{n} f(x) \mathrm{d} x\right)^{2} \leqslant \hat{M}_{n, 1} \int_{a}^{b}\left(f^{\prime}(x)\right)^{2} \mathrm{~d} x
$$

with the equality holding if and only if $f(x)=f(b)-$ $f(b)\left(\frac{x-b}{a-b}\right)^{n+2}$, where $\hat{M}_{n, 1}=\frac{(b-a)^{2 n+3}}{(n+1)^{2}(2 n+3)}$.

Corollary 5 Suppose $f(x) \in C^{2}[a, b]$ and $f(a)=$ $f^{\prime}(a)=0$, then for any $n \in \mathbb{N}$,

$$
\left(\int_{a}^{b}(x-b)^{n} f(x) \mathrm{d} x\right)^{2} \leqslant \hat{M}_{n, 2} \int_{a}^{b}\left(f^{\prime \prime}(x)\right)^{2} \mathrm{~d} x,
$$

with the equality holding if and only if

$$
f(x)=\frac{f(b)}{n+3}\left(\frac{x-b}{a-b}\right)^{n+4}+f(b)\left(\frac{a-x}{a-b}-\frac{1}{n+3} \frac{x-b}{a-b}\right),
$$

where $\hat{M}_{n, 2}=(b-a)^{2 n+5} /(n+1)^{2}(n+2)^{2}(2 n+5)$.

The inequalities related to $\left(\int_{a}^{b}\left(x-\frac{a+b}{2}\right)^{n} f(x) \mathrm{d} x\right)^{2}$ and $\int_{a}^{b}\left(f^{(k)}(x)\right)^{2} \mathrm{~d} x$

Theorem 7 Suppose $f(x) \in C^{1}[a, b], f(a)=f(b)$ and set $c=\frac{a+b}{2}$, then for any odd number $n$,

$$
\left(\int_{a}^{b}(x-c)^{n} f(x) \mathrm{d} x\right)^{2} \leqslant \bar{M}_{n, 1} \int_{a}^{b}\left(f^{\prime}(x)\right)^{2} \mathrm{~d} x,
$$

with equality holding if and only if

$$
f(x)=(f(a)-f(c))\left(\frac{x-c}{a-c}\right)^{n+2}+f(c),
$$

where $\bar{M}_{n, 1}=(b-a)^{2 n+3} /(n+1)^{2}(2 n+3) 2^{2 n+2}$.

Proof: Using the integration by parts formula, and noticing the given condition $f(a)=f(b)$, one may obtain

$$
\int_{a}^{b}(x-c)^{n} f(x) \mathrm{d} x=-\frac{1}{n+1} \int_{a}^{b}(x-c)^{n+1} f^{\prime}(x) \mathrm{d} x .
$$

According to the Cauchy-Schwarz integral inequal- 
ity, it follows

$$
\begin{aligned}
\left(\int_{a}^{b}(x-c)^{n} f(x) \mathrm{d} x\right)^{2} & =\left(-\frac{1}{n+1} \int_{a}^{b}(x-c)^{n+1} f^{\prime}(x) \mathrm{d} x\right)^{2} \\
& \leqslant \frac{\int_{a}^{b}(x-c)^{2(n+1)} \mathrm{d} x}{(n+1)^{2}} \int_{a}^{b}\left(f^{\prime}(x)\right)^{2} \mathrm{~d} x \\
& =\frac{(b-a)^{2 n+3}}{(n+1)^{2}(2 n+3) 2^{2 n+2}} \int_{a}^{b}\left(f^{\prime}(x)\right)^{2} \mathrm{~d} x \\
& =\bar{M}_{n, 1} \int_{a}^{b}\left(f^{\prime}(x)\right)^{2} \mathrm{~d} x
\end{aligned}
$$

where $\bar{M}_{n, 1}$ is defined in Theorem 7. Likewise, the equality holds in (8) if and only if $f^{\prime}(x)=\mu(x-$ c) ${ }^{n+1}$, i.e., $f(x)=\frac{\mu}{n+2}(x-c)^{n+2}+C$. Noting that $f(c)=C$ and $f(a)=f(b)$ one may get $f(x)=$ $f(c)+(f(a)-f(c))\left(\frac{x-c}{a-c}\right)^{n+2}$, and this completes the proof.

Remark 4 The condition $f(a)=f(b)$ and $n$ is an odd number in Theorem 7 can be replaced by $f(a)=$ $f(b)=0$ and $n \in \mathbb{N}$, while the result remains the same.

Remark 5 Setting $n=1$ and $f(a)=f(b)$ in Theorem 7 , it holds

$$
\left(\int_{a}^{b}\left(x-\frac{a+b}{2}\right)^{n} f(x) \mathrm{d} x\right)^{2} \leqslant \frac{(b-a)^{5}}{320} \int_{a}^{b}\left(f^{\prime}(x)\right)^{2} \mathrm{~d} x .
$$

The extension of Theorem 7 is given by the following result.

Theorem 8 Suppose $f(x) \in C^{k}[a, b], k \geqslant 1$, and $f^{(l)}(a)=f^{(l)}(b)=0(l=0,1, \ldots, k-1)$. Let $c=\frac{a+b}{2}$, then for any $n \in \mathbb{N}$,

$$
\left(\int_{a}^{b}(x-c)^{n} f(x) \mathrm{d} x\right)^{2} \leqslant \bar{M}_{n, k} \int_{a}^{b}\left(f^{(k)}(x)\right)^{2} \mathrm{~d} x,
$$

with the equality holding if and only if

$$
\begin{aligned}
f^{(k)}(x) & =\mu(x-c)^{n+k}, \\
f^{(l)}(a) & =f^{(l)}(b)=0, \quad l=0,1, \ldots, k-1,
\end{aligned}
$$

where $\mu$ is an arbitrary constant, and

$$
\bar{M}_{n, k}=\frac{(b-a)^{2 n+2 k+1}}{(n+1)^{2}(n+2)^{2} \cdots(n+k)^{2}(2 n+2 k+1) 2^{2 n+2 k}} .
$$

\section{CONCLUSION}

In this work, we investigated the methods of how to construct new integral inequalities. By using the Cauchy-Schwarz inequality and the formula of integration by parts, we obtained several classes of new inequalities which even contain parameters in them. Moreover, the optimal constant factors for the proposed inequalities are discussed and carried out. Those techniques used in this paper can also be applied to the construction of new inequalities in multiple integrals, and this will be the focus of our further work.

Acknowledgements: This work is partially supported by the National Natural Science Foundation of China (No. 11861039), Jiangxi Province Postgraduate Quality Course and Teaching Case Project (No. 2020-36) , and the Teaching Reform Project of Nanchang Hangkong University (No. JY1937).

\section{REFERENCES}

1. Hardy G, Littlewood JE, Pólya G (1952) Inequalities, 2nd edn, Cambridge University Press, Cambridge, UK.

2. Mitrinovic DS, Vasic PM (1970) Analytic Inequalities, Springer-Verlag, Berlin, Heidelberg.

3. Wang W-S, Shen C-X (2008) On a generalized retarded integral inequality with two variables. $J$ Inequal Appl 2008, ID 518646.

4. Li M, Zhao Y-L (2018) A fast energy conserving finite element method for the nonlinear fractional Schrödinger equation with wave operator. Appl Math Comput 338, 758-773.

5. Li M (2019) A high-order split-step finite difference method for the system of the space fractional CNLS. Eur Phys J Plus 134, ID 244.

6. Zhang Z, Li M, Wang Z (2019) A linearized CrankNicolson Garlerkin FEMs for the nonlinear fractional Ginzburg-Landau equation. Appl Anal 98, 2648-2667.

7. Yang Q, Gao M (2005) A new extension on HardyHilbert's integral inequality and its application. Pure Appl Math 21, 91-98.

8. Gu X-M, Huang T-Z, Xu W-R, Li H-B, Li L, Zhao X-L (2014) Some refinements and generalizations of I. Schur type inequalities. Sci World $J$ 2014, ID 709358.

9. Li Y, Gu X-M, Xiao J (2015) A note on the proofs of generalized Radon inequality. Math Morav 22, 59-67.

10. Li Y, Gu X-M, Zhao J (2018) The weighted arithmetic mean-geometric mean inequality is equivalent to the Hölder inequality. Symmetry 10, ID 380. 